\title{
DETERMINATION OF REACTION FORCES AT KINEMATICAL JOINTS
}

\author{
DO SANH \\ Hanoi University of Technology, Hanoi
}

\begin{abstract}
The determination of reaction forces at kinematical joints of mechanism plays an important role in dynamics of machines. In the paper a method of determining the reaction at kinematical joints is presented.

It is established a set of equations that are divided into two groups. The one describes the motion of the system and the other allows to calculate the reaction at kinematical joints.
\end{abstract}

\section{Introduction}

The determination of reaction forces at kinematical joints has investigated in many works. In engineering the D'Alembert's method is usually applied for calculating the reaction forces at kinematical joints. However, such a method depends on each concrete structure of mechanisms.

There was an idea of making a general method for the problem. That is to apply the method of analytical mechanics for determining reaction forces at kinematical joints of a chain of links [7]. However, such method hasn't yet taken clearness and simplicity from engineering point of view.

\section{Equations of motion of a constrained mechanical system}

Let us consider a holonomic mechanical system of $n$ degrees of freedom. Denote generalized coordinates of the system under consideration by $q_{i}(i=\overline{1, n})$.

Assume that the matrix of inertia of the system is denoted by $\mathbf{A}$, which is an $n \times n$ positive define symmetric matrix. The elements of the matrix $\mathbf{A}$ depend on generalized coordinates, i.e. $\mathbf{A}=\mathbf{A}(\mathbf{q})$; where $\mathbf{q}$ is an $n \times 1$ matrix of generalized coordinates, that is:

$$
\mathbf{q}^{T}=\left\|q_{1} q_{2} \quad q_{3} \quad \ldots q_{n}\right\|
$$

the notation $T$ denotes a transpose of matrix. 
Suppose that the expression of kinetic energy of the system is written in the form:

$$
T=\frac{1}{2} \dot{\mathbf{q}}^{T} \mathbf{A} \dot{\mathbf{q}}
$$

where $\dot{q}$ is an $n \times 1$ matrix of generalized velocities, $\dot{\mathbf{q}}$ is the transpose of the matrix of $\dot{\mathbf{q}}$, i.e.:

$$
\dot{\mathbf{q}}^{T}=\left\|\dot{q}_{1} \dot{q}_{2} \dot{q}_{3} \ldots \dot{q}_{n}\right\|
$$

Denote the generalized forces of applied ones by $Q_{i}(t, q, \dot{q})$ and $\mathbf{Q}$ is their matrix notation, that is:

$$
\mathrm{Q}^{T}=\left\|Q_{1} Q_{2} Q_{3} \ldots Q_{n}\right\|
$$

Suppose that the system under consideration is subjected to constraints of the form:

$$
f_{\alpha}\left(t, q_{1}, q_{2}, q_{3}, \ldots, q_{n}\right)=0 ; \quad \alpha=\overline{1, r}
$$

Basing on the principle of compatibility [2-5] the motion of a constrained system must be written in the form:

$$
\mathbf{A} \ddot{\mathbf{q}}=\mathbf{Q}+\mathbf{G}+\mathbf{R}
$$

where $\mathbf{G}$ is an $n \times 1$ matrix, which consists of the elements of the matrix of inertia $\mathbf{A}$, but $\mathbf{R}$ is the reaction matrix which must satisfy the condition of ideality of the constraints (2.5). As known [3-5] this condition is

$$
\mathbf{D} \mathbf{R}=\mathbf{O},
$$

where $\mathbf{D}$ is $(n-r) \times n$ matrix, its elements are just coefficients in expressions of generalized accelerations written in the terms of independent generalized accelerations by solving the constraint equations (2.5).

By means of the equations (2.6) and (2.7) it is possible to obtain a set of equations, that are divided into two groups, one of these group describes motion of the system under consideration and the other group gives the reactions of constraints.

\section{Algorithm for calculating reactions at kinematical joints}

To determine the reactions at the kinematical joint let us release just that joint. The action of the released joint on the system is replaced by the reaction forces. In other words, the freed system is then assumed to be that without constraints under actions of applied and reaction forces. 
Because of releasing constraints, the number of degrees of freedom of the freed system is bigger than the original system.

Adapting to this let us introduce $r$ new coordinates denoted by $q_{n+1}, q_{n+2}, \ldots$, $q_{n+r}$. The number of new coordinates is equal to the increased number of degrees of freedom. In order that the motion of the freed system is identical to the original system, it is necessary to add some conditions. Such conditions play the role of constraints. The reactions of these constraints are just the reactions of the remarked joints.

Example. As an illustration let us consider a hammer crush machine shown in Fig. 1. The drum is a homogeneous disk of radius $R$ and moment of inertia $I_{1}$ about the rotation axis $O$.

There is a couple of force $M$ acting on the drum. The physical pendulum has the mass $m$ and the moment of inertia $I_{2}$ about its center of mass $C_{2}\left(A C_{2}=a\right)$. The physical pendulum is hung up against the disk at the joint A. Determine the reaction forces at the articulated joint $A$ and write the equations of motion of the hammer crush machine.

Consider the hammer crush machine as a system of two degrees of freedom. Let choose generalized coordinates as $\varphi$ and $\theta$ - the angle coordinates of the disk and physical pendulum respectively. These angles are formed by $\mathrm{OA}$ and $\mathrm{AC}_{2}$ with verticals. The system of hammer crush machine under consideration is called the original system. To calculate the reactions at joint $A$ let release the physical pendulum from the drum (see Fig. 2).

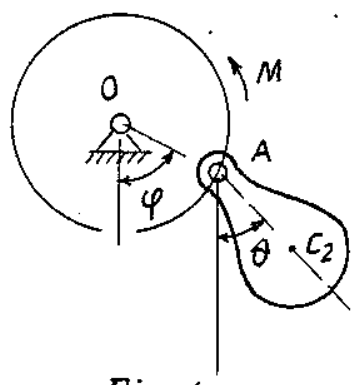

Fig. 1

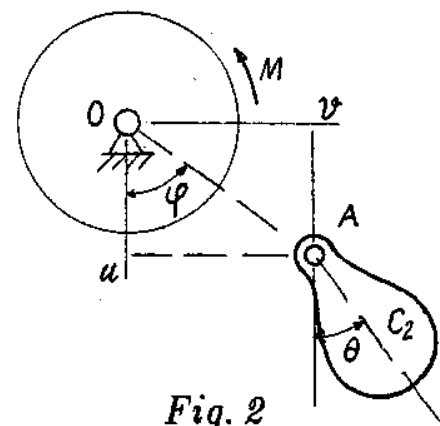

Fig. 2

The freed system has 4 degrees of freedom. Let us choose generalized coordinates of this system as $\varphi, \theta, u, v$, where $\varphi, \theta$ have the same meaning as above, but $u, v$ are rectangular coordinates of the joint A located on the physical pendulum. In order that the motion of the freed system is identical to the original system it is necessary to use the following conditions

$$
u-R \cos \varphi=0, \quad v-R \sin \varphi=0
$$


These conditions are treated as constraints imposed on the freed system.

By putting:

$$
\sigma=u-R \cos \varphi, \quad \xi=v-R \sin \varphi
$$

we have

$$
u=\sigma+R \cos \varphi, \quad v=\xi+R \sin \varphi
$$

Instead of $u, v$, we use the variables $\sigma, \xi$. Equations of constraints (3.1) are now:

$$
\sigma=0 ; \quad \xi=0
$$

The matrix of inertia of the freed system is

$$
\mathbf{A}=\left\|\begin{array}{cccc}
I_{1}^{0} & m a R \cos (\varphi-\theta) & -m R \sin \varphi & m R \cos \varphi \\
m a R \cos (\varphi-\theta) & I_{2}^{0} & -m a \sin \theta & m \\
-m R \sin \varphi & -m a \sin \theta & m & 0 \\
m R \cos \varphi & m a \cos \theta & 0 & m
\end{array}\right\|
$$

where

$$
I_{1}^{0}=I_{1}+m R^{2} ; \quad I_{2}^{0}=I_{2}+m a^{2}
$$

Notice that the $2 \times 2$ first matrix $A_{0}$ on the principal diagonal of the matrix $\mathbf{A}$ is the matrix of inertia of the original system:

$$
\mathbf{A}_{0}=\left\|\begin{array}{cc}
I_{1}^{0} & \operatorname{maR} \cos (\varphi-\theta) \\
\operatorname{maR} \cos (\varphi-\theta) & I_{2}^{0}
\end{array}\right\| .
$$

In accordance with the constraints (3.4) the matrix $D$ in (2.7) is a $2 \times 4$ matrix, which has the form:

$$
\mathbf{D}=\left\|\begin{array}{llll}
1 & 0 & 0 & 0 \\
0 & 1 & 0 & 0
\end{array}\right\|
$$

The matrix $\mathrm{G}$ in (2.6) will be now:

$$
\mathbf{G}=\left\|\begin{array}{c}
-m R a \sin (\varphi-\theta) \dot{\theta}^{2} \\
m R a \sin (\varphi-\theta) \dot{\varphi}^{2} \\
m R \cos \varphi \dot{\varphi}^{2}+m a \cos \theta \dot{\theta}^{2} \\
m R \sin \varphi \dot{\varphi}^{2}+m a \sin \theta \dot{\theta}^{2}
\end{array}\right\|
$$

The matrix of applied forces is of the form:

$$
\mathbf{Q}=\left\|\begin{array}{c}
M-m g \sin \varphi \\
-m g a \sin \theta \\
m g \\
0
\end{array}\right\|
$$


The condition of ideality of the constraints by (2.7) is written as follows:

$$
\left\|\begin{array}{cccc}
1 & 0 & 0 & 0 \\
0 & 1 & 0 & 0
\end{array}\right\|\left\|\begin{array}{l}
R_{\varphi} \\
R_{\theta} \\
R_{\sigma} \\
R_{\xi}
\end{array}\right\|=0 .
$$

From here we obtain:

$$
R_{\varphi}=0, \quad R_{\theta}=0
$$

Therefore we can write

$$
\mathbf{R}^{T}=\left\|0 \quad 0 \quad R_{\sigma} \quad R_{\xi}\right\|
$$

Because of (3.4) we have

$$
\sigma=0, \quad \xi=0, \quad \dot{\sigma}=0, \quad \dot{\xi}=0, \quad \ddot{\sigma}=0, \quad, \ddot{\xi}=0 .
$$

Thus the matrix $\ddot{\mathbf{q}}$ will take the following form:

$$
\ddot{\mathbf{q}}^{T}=\|\ddot{\varphi} \quad \ddot{\theta} \quad 0 \quad 0\| .
$$

Equation (2.6) is now:

$$
\begin{aligned}
& \left\|\begin{array}{cccc}
I_{1}^{0} & m a R \cos (\varphi-\theta) & -m R \sin \varphi & m R \cos \varphi \\
m a R \cos (\varphi-\theta) & I_{2}^{0} & -m a \sin \theta & m \\
-m R \sin \varphi & -m a \sin \theta & m & 0 \\
m R \cos \varphi & m a \cos \theta & 0 & m
\end{array}\right\| \| \\
& =\left\|\begin{array}{c}
M-m g R \sin \varphi-R a \sin (\varphi-\theta) \dot{\theta}^{2} \\
-m g a \sin \theta+m R a \sin (\varphi-\theta) \dot{\varphi}^{2} \\
m g+m R \cos \varphi \dot{\varphi}^{2}+m a \cos \theta \dot{\theta}^{2} \\
m R \sin \varphi \dot{\varphi}^{2}+m a \sin \theta \dot{\theta}^{2}
\end{array}\right\|+\left\|\begin{array}{c}
0 \\
0 \\
R_{\sigma} \\
R_{\xi}
\end{array}\right\| .
\end{aligned}
$$

The matrix equation (3.16) can be written in the form of two matrix equations:

$$
\begin{aligned}
& \left\|\begin{array}{cc}
I_{1}^{0} & m a R \cos (\varphi-\theta) \\
m a R \cos (\varphi-\theta) & I_{2}^{0}
\end{array}\right\|\left\|\begin{array}{c}
\ddot{\varphi} \\
\ddot{\theta}
\end{array}\right\|= \\
& =\left\|\begin{array}{c}
M-m g R \sin \varphi-m a R \sin (\varphi-\theta) \dot{\theta}^{2} \\
-m g a \sin \theta+m a R \sin (\varphi-\theta) \dot{\varphi}^{2}
\end{array}\right\|
\end{aligned}
$$


and

$$
\left\|\begin{array}{l}
R_{\sigma} \\
R_{\xi}
\end{array}\right\|=\left\|\begin{array}{cc}
-m R \sin \varphi & -m a \sin \theta \\
m R \cos \varphi & m a \cos \theta
\end{array}\right\|\left\|\begin{array}{c}
\ddot{\varphi} \\
\ddot{\theta}
\end{array}\right\|-\left\|\begin{array}{c}
m g+m R \cos \varphi \dot{\varphi}^{2}+m a \cos \theta \dot{\theta}^{2} \\
m R \sin \varphi \dot{\varphi}^{2}+m a \sin \theta \dot{\theta}^{2}
\end{array}\right\| .
$$

Equations (3.17) describe the motion of the original system - the hammer crush machine, but the reactions at the joint $A$ are drawn from the equations (3.18). By such a way we obtain two groups of equations. The one describes the motion of the original system, the other gives the reactions at the mentioned joint.

Notice that (3.9) and (3.10) can be written in the form:

$$
\mathbf{G}=\left\|\begin{array}{c}
\mathbf{G}_{0} \\
\ldots \ldots \ldots \ldots \ldots \\
m R \cos \varphi \dot{\varphi}^{2}+m a \cos \theta \dot{\theta}^{2} \\
m R \sin \varphi \dot{\varphi}^{2}+m a \sin \theta \dot{\theta}^{2}
\end{array}\right\|, \quad \mathbf{Q}=\left\|\begin{array}{c}
\mathbf{Q}_{0} \\
\ldots \ldots \\
m g \\
0
\end{array}\right\|
$$

where $\mathbf{G}_{0}$ and $\mathbf{Q}_{0}$ are the $2 \times 1$ matrices

$$
\mathbf{G}_{0}=\left\|\begin{array}{c}
m R a \sin (\varphi-\theta) \dot{\theta}^{2} \\
m R a \cos (\varphi-\theta) \dot{\varphi}^{2}
\end{array}\right\|, \quad \mathbf{Q}_{0}=\left\|\begin{array}{c}
M-m g r \sin \varphi \\
m g a \sin \theta
\end{array}\right\|
$$

It is easy to see that $\mathbf{G}_{0}$ is just the matrix $\mathbf{G}$ of the original system, but $\mathbf{Q}_{0}$ is the matrix of applied forces acting on the original system. In other words, the matrices $\mathbf{G}_{0}$ and $\mathbf{Q}_{0}$ are calculated for the original system, while $\mathbf{G}$ and $\mathbf{Q}$ are calculated for the freed system.

By such a way, the equation (3.17) can be written as follows:

$$
\mathbf{A}_{0} \ddot{\mathbf{q}}_{0}=\mathbf{G}_{0}+\mathbf{Q}_{0}
$$

where $\ddot{\mathbf{q}}_{0}$ is an $n \times 1$ matrix of generalized accelerations of the original system, i.e.

$$
\ddot{\mathbf{q}}_{0}^{T}=\|\ddot{\varphi} \ddot{\theta}\| .
$$

Notice that $\mathbf{A}_{0}$ is the $n \times n$ matrix, which is made from $n$ first rows and columns of the matrix $\mathbf{A}$. In other words, $\mathbf{A}_{0}$ is at the first left corner and on principal diagonal of the matrix $\mathbf{A}$.

We construct now an $r \times n$ matrix denoted by $\mathbf{B}$. This matrix is made from $r$ last rows and $n$ first columns of the matrix $\mathbf{A}$. In the above example, the matrix $\mathbf{B}$ is of the form:

$$
\mathbf{B}=\left\|\begin{array}{cc}
-m R \sin \varphi & -m a \sin \theta \\
m R \cos \varphi & m a \cos \theta
\end{array}\right\|
$$


Next we introduce the $r \times 1$ matrices, $\mathbf{G}_{1}$ and $\mathbf{Q}_{1}$, that are made from last $r$ rows of the matrices $\mathbf{G}$ and $Q$ respectively.

In accordance with the above example we have:

$$
\mathrm{G}_{1}=\left\|\begin{array}{cc}
m R \cos \varphi \ddot{\varphi}^{2}+m a \cos \theta & \dot{\theta}^{2} \\
m R \sin \varphi \dot{\varphi}^{2}+m a \sin \theta \dot{\theta}^{2}
\end{array}\right\|, \quad \mathbf{Q}_{1}=\left\|\begin{array}{c}
m g \\
0
\end{array}\right\|
$$

Besides we denote:

$$
\mathbf{R}_{1}^{T}=\left\|R_{\sigma} \quad R_{\xi}\right\|
$$

Notice that the $n \times 1$ matrix $\mathbf{R}_{1}$ is made from a last rows of the matrix of reactions $\mathbf{R}$. Equations (3.18) can be written then:

$$
\mathbf{R}_{1}=\mathbf{B} \ddot{\mathbf{q}}_{0}-\mathbf{G}_{1}-\mathbf{Q}_{1} \text {. }
$$

By such a way we obtain two groups of equations, the equation (3.21) without reactions describes the motion of the system, but equations (3.26) gives the reaction at the joint under consideration.

Because of positive definiteness of $A_{0}$, there is the inverse matrix $A_{0}^{-1}$. Thus, from (3.21) we have

$$
\ddot{\mathbf{q}}_{0}=A_{0}^{-1}\left(\mathbf{G}_{0}+\mathbf{Q}_{0}\right) \text {. }
$$

Substituting (3.21) into (3.26) we can calculate the reactions at the joint, that is

$$
\mathbf{R}_{1}=\mathbf{B} A_{0}^{-1}\left(G_{0}+Q_{0}\right)-G_{1}-Q_{1} .
$$

It is important that the reactions calculated by means of (3.28), don't contain accelerations. Go bask to the above example. It is easy to calculate the inverse of matrix $\mathbf{A}_{0}$ :

$$
\mathbf{A}_{0}^{-1}=\left\|\begin{array}{cc}
I_{2}^{0} & -m a R \cos (\varphi-\theta) \\
-m a R \cos (\varphi-\theta) & I_{1}^{0}
\end{array}\right\|,
$$

where

$$
\Delta=I_{1}^{0} I_{2}^{0}-m^{2} a^{2} R^{2} \cos ^{2}(\theta-\varphi)
$$

Therefore

$$
\begin{aligned}
& \mathbf{B} \mathbf{A}_{0}^{-1}=\frac{1}{\Delta} \times \\
& \left\|\begin{array}{ll}
m R\left[m a^{2} \sin \theta \cos (\theta-\varphi)-I_{2}^{0} \sin \varphi\right] & m a\left[m R^{2} \sin \varphi \cos (\theta-\varphi)-I_{1}^{0} \sin \theta\right] \\
m R\left[I_{2}^{0} \cos \varphi-m a^{2} \cos \theta \cos (\theta-\varphi)\right] & m a\left[I_{1}^{0} \cos \theta-m R^{2} \cos \varphi \cos (\theta-\varphi)\right]
\end{array}\right\|
\end{aligned}
$$


We have then

$$
\begin{aligned}
& \left\|\begin{array}{c}
R_{\sigma} \\
R_{\xi}
\end{array}\right\|= \\
& \left\|\begin{array}{cc}
m R\left[m a^{2} \sin \theta \cos (\theta-\varphi)-I_{2}^{0} \sin \varphi\right] & m a\left[m R^{2} \sin \varphi \cos (\theta-\varphi)-I_{1}^{0} \sin \theta\right] \\
m R\left[I_{2}^{0} \cos \varphi-m a^{2} \cos \theta \cos (\theta-\varphi)\right] & m a\left[I_{1}^{0} \cos \theta-m R^{2} \cos \varphi \cos (\theta-\varphi)\right]
\end{array}\right\| \\
& \times\left\|\begin{array}{c}
M-m g R \sin \varphi \\
m g a \cos \theta
\end{array}\right\|-\left\|\begin{array}{c}
m R \cos \varphi \dot{\varphi}^{2}+m a \cos \theta \dot{\theta}^{2}+m g \\
m R \sin \varphi \dot{\varphi}^{2}+m a \sin \theta \dot{\theta}^{2}
\end{array}\right\|
\end{aligned}
$$

It is easily to see that $R_{\sigma}, R_{\xi}$ are just vertical and horizontal components of the reaction at the kinematical joint $A$, respectively.

\section{Conclusions}

By means of equations (3.28) the reactions are determined independently to establishment of equations of motion. Of course, to do this it is necessary to calculate the inverse matrix of inertia as in the case of applying directly the principle of compatibility [2-5]. However, it is important that the dimension of the matrix of inertia of the original system is smaller than the freed system. This reduces remarkably the calculations.

The equations obtained are written in matrix forms. This is very appropriate for programming by means of $\mathrm{PC}$, especially, for using symbolic method. This publication is completed with the financial support from the Council for Natural Science of Vietnam.

\section{References}

1. Beghin H. Etude theoretique des compas gyrostatiques, Anschutz et Spary 1727, Paris, 1922.

2. Do Sanh. On the Principle of Compatibility and the equations of motion of a constrained mechanical system, ZAMM, 4, Berlin, 1980.

3. Do Sanh. On the motions of controlled mechanical system, Advanced in Mechanics, Vol. 7, No2 Warsaw, 1984.

4. Do Sanh. On the motion of constrained system. The thesis of Doctor of Science, Hanoi University of Technology, Hanoi 1984.

5. Do Sanh. A form of equations of motion of a constrained mechanical system, Proceedings of International Conference of Applied Dynamics, Hanoi 1995. 
6. Gutowski R., Radziszewski B. On the exact control problem of the mechanical system with program constraints problems of Nonlinear Vibration, 10, Warsaw 1969.

7. Lurie A. I. Analytical Mechanics, Publ. Non-Linear, Nauka, Moscow 1961.

8. Olas A. Synthesis of system with prescribed trajectories, Problems of NonLinear Vibration, 16, Warsaw 1975.

9. Xu M., Liu C. and Huston R. L. Analysis of Non-Linearly Constrained NonHolonomic Multibody System, International Journal of Non-Linear Mechanics, Vol. 25, No 5, 1990.

Received June 1, 1998

\section{XÁC ĐỊNH CÁC PHÂN LỰC TẠI CÁC KHỚP ĐộNG}

Việc xác định các phản lực tại các khớp động của cơ cấu có vai trò quan trọng trong bài toán động lực học của máy và cơ cấu.

Trong bài báo đưa ra một phương pháp tiện lợi cho việc xác định các phản lực khớp động. Đã thành lập hai nhóm phương trình: một nhóm cho phép xác định chuyển động của cơ cấu (phương trình chuyển động), còn một nhóm cho phép tính toán các phản lực tại các khớp động. 